\title{
Protocol Based Treatment in Pediatric Intensive Care Units
}

\author{
Rakesh Lodha $\cdot$ Sushil Kumar Kabra
}

Received: 5 October 2010 / Accepted: 5 October 2010 /Published online: 13 October 2010

(C) Dr. K C Chaudhuri Foundation 2010

Pediatric specialties are still in infancy in India and other developing countries. Pediatric intensive care is an important emerging specialty. In India, few centers in government and private hospitals have developed separate Pediatric Intensive Care Units (PICU). The care of critically ill patients has become increasingly complex as severity of illness continues to increase, the amount of clinical information available at the bedside is growing, and also the quantity of evidence supporting or refuting specific therapies and interventions for this population is escalating [1]. To ensure that the critically ill children receive the highest quality of care, various strategies are used; one of these is use of protocols for management of various conditions.

Modern equipments play an important role in the management and outcome of children. Equally important is the role of well trained staff working in the PICUs. Training of doctors, nurses and other support staff is a very important component of intensive care in any specialty including pediatrics. For delivery of quality care in the PICUs there is need for regular training of residents and nurses. A protocol based approach is not only useful in training but also ensures same standards of care even if new resident/nurse is posted in PICU.

With the availability of modern technology, PICU care has improved but the cost has increased significantly. Some

\section{R. Lodha $(\bowtie) \cdot$ S. K. Kabra}

Division of Pediatric Pulmonology and Intensive Care,

Department of Pediatrics, All India Institute of Medical Sciences, New Delhi, India

e-mail: rakesh_lodha@hotmail.com newer technologies may not be available or not be very cost effective; it is important to develop management protocols depending on the available resources and cost efficacy. Protocols are available from developed countries, which may not be followed as such in India as the type of illness, severity and priority may be different. Therefore, it is important that each PICU develops its own protocols based on available information, patient profile, priorities and available resources.

It is desirable to objectively document the effect of implementation of various protocols in the PICU. For this, we need to collect the baseline data prior to protocol implementation. This is followed by collection of data on the process of care measures and the patient-based outcomes [1]. Based on these outcomes, the protocols should be reviewed and modified if required.

At All India Institute of Medical Sciences, New Delhi, a separate PICU was started in 1986. Initially, a six bed unit was started. In the initial years, the common indications for admission were septicemia, lower respiratory tract infections and CNS infections [2]. The numbers of children mechanically ventilated gradually increased from $1 \%$ to $35 \%$ [2]; the mortality rate was $23.5 \%$. Over the years, there was a steady improvement in the services; better equipments are now available and more staff-pediatricians, nurses have joined. Currently, severe sepsis, septic shock and respiratory illnesses are the major indications for admission to PICU [3]. Nearly $90 \%$ children are mechanically ventilated and the predicted mortality (based on PRISM, PIM) is approximately $25 \%$. As compared to the PICUs in the developed countries [4] and also with PICUs in the private sector [5], the average severity of illness in 
children admitted to the PICU here is higher; there may be multiple reasons for the same [3].

To improve the care of critically ill children, PICU has developed protocol based treatment over past 10 year; the protocols were initially prepared by residents, discussed and put into practice. These have been revised regularly. From this issue of IJP, we are publishing protocols followed in our PICU with an aim to provide basic information for others who are planning to start or are running PICU services. As we discussed above, the protocol should be adapted considering the patient profile, priorities of the unit and available resources. These protocols may be modified as per the unit's needs. The protocols are for common conditions being treated in PICUs along with those for supportive care.

\section{References}

1. Kollef MH, Micek ST. Using protocols to improve patient outcomes in the intensive care unit: focus on mechanical ventilation and sepsis. Semin Respir Crit Care Med. 2010;31:19-30.

2. Kapil D, Bagga A. The profile and outcome of patients admitted to a pediatric intensive care unit. Indian J Pediatr. 1993;60:5-10.

3. Thukral A, Lodha R, Irshad M, Arora NK. Performance of Pediatric Risk of Mortality (PRISM), Pediatric Index of Mortality (PIM), and PIM2 in a pediatric intensive care unit in a developing country. Pediatr Crit Care Med. 2006;7:356-61.

4. Namachivayam P, Shann F, Shekerdemian L, et al. Three decades of pediatric intensive care: Who was admitted, what happened in intensive care, and what happened afterward. Pediatr Crit Care Med. 2010;11:549-55.

5. Khilnani P, Sarma D, Singh R, et al. Demographic profile and outcome analysis of a tertiary level pediatric intensive care unit. Indian J Pediatr. 2004;71:587-91. 